\title{
НЕОДНОРОДНЫЕ УРАВНЕНИЯ СВЕРТКИ
}

В. В. НАПАЛКОВ

Отдел Физики и Математики Баикирского Филиала АН СССP ул. Тукаева 50, SU-450057 У $6 a$, CCCP

В работе рассматриваются неоднородные уравнения свертқи в полупространствах комплексного пространства $C^{n}$. Найдены условия разрешимости таких уравненхй. В частности, для размерности $n=1$ эти условия состоят в том, чтобы характеристическая функщия уравнения свертки имела вполне регулярный рост.

\section{1. Введение}

Пусть $\mathscr{D} \subset C^{n}$ - произвольная область; $H(\mathscr{D})$ - пространство функций, аналитических в $\mathscr{D}$, с топологией равномерной сходимости на компактах из $\mathscr{D}$; $H^{*}(\mathscr{D})$ - сопряженное с $H(\mathscr{D})$ пространство с топологией равномерной сходимости на ограниченных множествах из $H(\mathscr{D})$. Так как $H(\mathscr{D})$ является замкнутым подпространством пространства всех непрерывных функций на $\mathscr{D}$ с топологией равномерной сходимости на компактах из $\mathscr{D}$, то всякий функционал $F \in H^{*}(\mathscr{D})$ определяется некоторой комплексной конечно-аддитивной мерой $\mu_{F}$, supp $\mu_{F} \subset \mathscr{D}$, т.е. $\langle F, f\rangle=\int f(z) d \mu_{F}, f(z) \in H(D)$. Пусть $F_{0} \in$ $\in H^{*}(\mathscr{D})$. Тогда функционал $F_{0}$ определяет в пространстве $H(\mathscr{D})$ следуюший оператор свертки

$$
M_{F_{0}}[f]=F_{0} * f \equiv\left\langle F_{0}, f(z+u)\right\rangle .
$$

Так как носитель меры $\mu_{F_{0}}$ компактно лежит в $\mathscr{D}$, то свертка $F_{0} * f$ является функцией, аналитичесқой в некоторой окрестности нуля. Характеристической функцией оператора (1) будем назыгвать функцию $\hat{F}_{0}(\lambda)=\left\langle F_{0}, \exp \langle\lambda, z\rangle\right\rangle$.

Для оператора (1) естественным образом возникают следующие две задачи:

1. Аппроксимационная задача - всякое ли решение однородного уравнения $M_{F_{0}}[f]=0$ из пространства $H(\mathscr{D})$ можно аппроксимировать линейными комбинациями экспоненциальных многочленов, удовлетворяющих этому же уравнению; 
2. Найти условия разрешимости неоднородного уравнения (2)

$$
M_{F_{0}}[f]=g .
$$

Первая задача изучалась в работах [1], [2], [3], [4], [5], [6], [7]. Бъило показано, что аптроксимационная задача имеет положительное решение в случаях, когда $\mathscr{D}=C^{n}([1],[2])$; далее, когда $\mathscr{D}$ - полупространство в $C^{n}$ или область ,типа полосы” ([5]). Наконец, в работах [6], [7] с помощью нового подхода аттроксимационная задача была решена, в частности, для произвольной трубчатой области в $C^{n}$.

Вторая задача изучалась Мальгранжем [1], хоторый доказал разрешимость уравнения (2), когда $\mathscr{D}=\boldsymbol{C}^{n}$. Разрешимость уравнения (2) была доказана Мартино [3] для произвольной выпуклой области $\mathscr{D} \subset C^{n}$, но при существенном ограничении на характеристическуюо функцию оператора $M_{F_{0}}$ она предполагалась функцией минимального типа. Результат Мартино был затем существенно обобщен В. В. Моржаковым [8], который нашел более общие достаточные условия разрешимости уравнения (2).

В данной работе мы находим общий критерий разрешимости уравнения (2) в случае, когда $\mathscr{D}-$ полупространство в $\boldsymbol{C}^{n}$. Используя этот критерий и то, что любая вытуклая область исчерпывается изнутри выпуклыми многогранниками, можно находить условия разрешимости уравнения (2) для выпукльх областей. Заметим, что в случае размерности $n=1$ отсюда получаются необходимые и достаточные условия разрешимости уравнения (2) для произвольной выпуклой области $\mathscr{D}$, которые ранее были найдены в работе [9].

\section{2. Предварительные сведения}

(a) Пусть $\mathscr{D}$ является областью Рунге. Введем оператор $T_{\mathscr{D}}$, действующий из пространства $H^{*}(\mathscr{D})$ в пространство целых функций экспоненциального типа по правилу : если $F \in H^{*}(\mathscr{D})$, то $T_{\mathscr{D}}[F]$ - преобразование Лапласса функционала $F$, то есть $T_{\mathscr{D}}[F]=\hat{F}(\lambda)=\langle F, \exp \langle\lambda, z\rangle\rangle$. Множество всех преобразований Лапласа элементов из $H^{*}(\mathscr{D})$ обозначим через $P_{\mathscr{D}}$. Так как $\mathscr{D}$ является областью Рунге, то отображение $T_{\mathscr{D}}$ устанавливает взаимно однозначное соответствие между множествами $H^{*}(\mathscr{D})$ и $P_{\mathscr{D}}$. Используя этот изоморфизм, наделим множество $P_{\mathscr{D}}$ топологией так, чтобы отображение $T_{\mathscr{D}}$ было топологическим изоморфизмом.

(б) Компактное множество $K \subset \mathscr{D}$ назыпается определятоиим функиионал $F \in H^{*}(\mathscr{D})$ (см. [10], стр. 135), если для всякой окрестности $\omega$ этого компакта существует константа $C_{\omega}$ такая', что

$$
|\langle F, f\rangle| \leqslant C_{\omega} \sup _{\omega}|f|, f \in H(\mathscr{D}) .
$$

Из определения топологии в $H(\mathscr{D})$ следует, что для любого функционала $F \in H^{*}(\mathscr{D})$ существует некоторый компакт, определягиий $F$.

Для произволного компакта $K \subset C^{n}$ положим $H_{K}(\zeta)=\sup _{z \in K}(\operatorname{Re}\langle z, \zeta\rangle)$.
Очевидно, $H_{K}(\zeta)$ - выпуклая положительно однородная функция переменной $\zeta$ и если компақт $K$ является выпуклым, то $K=\left\{z \in C^{n}:(\operatorname{Re}\langle z, \zeta\rangle) \leqslant\right.$ $\left.\leqslant H_{K}(\zeta), \zeta \in C^{n}\right\}$. Имеет место следующая

Tеорема А (см. [10], стр. 137). Если функционал $F \in H^{*}(\mathscr{D})$ определяется компактным множеством $K$, то для каждого $\delta>0$ существует постоянная $C_{\delta}$ такая, что

$$
|\langle F, \exp \langle z, u\rangle\rangle| \leqslant C_{\delta} \cdot \exp \left(H_{K}(z)+\delta \cdot|z|\right), \quad z \in C^{n} .
$$

Обратно, если $K \subset \mathscr{D}$ - выпуклое компактное множество и $\varphi(z)$-уелая функиия, удовлетворяюияая неравенству (3) для всякого $\delta>0$, то существует функчионал $F \in H^{*}(\mathscr{D})$, определяемый компактом $K$ такой, что $\varphi(z)=$ $=\langle F, \exp \langle z, u\rangle\rangle$.

\section{3. Решение неоднородных уравнений свертки в полупространстве}

Пусть $\varphi(z)$ - целая функция экспоненциального типа в $C^{n}$. Введем две функции

$$
L_{\varphi}(z)=\varlimsup_{r \rightarrow \infty} \frac{1}{r} \ln |\varphi(r \cdot z)|, \quad L_{\varphi}^{*}(z)=\varlimsup_{\zeta \rightarrow z} L_{\varphi}(z), \quad z \in C^{n},
$$

которые будем называть, соответственно, радиальным и регуляризованным радиальным индикатором. Отметим несколько нужньгх нам для дальнейшего свойств функций $L_{\varphi}(z)$ и $L_{\varphi}^{*}(z)$ (см., например, [11], стр. 286).

1) Функция $L_{\varphi}^{*}(z)$ является положительно однородной порядка $\varrho=1$ и плюрисубгармонической функцией в $C^{n}$.

2) Множество точек $z$, для которьх $L_{\varphi}(z) \neq L_{\varphi}^{*}(z)$ имеет нулевую меру Лебега.

3) Для всякого $z^{0} \in C^{n},\left|z^{0}\right|=1$ и любого числа $A>L_{\varphi}^{*}\left(z^{0}\right)$ существует такой открытый конус $V \subset C^{n}$ с вершиной в начале координат, что $z^{0} \in V$ и при всех $z \in V$ справедливо неравенство: $\ln |\varphi(z)| \leqslant A|z|$.

Пусть $F \in H^{*}\left(C^{n}\right)$. Найдем преобразование Лапласа $\hat{F}(\lambda)$ функционала $F$ и построим функции $L_{\hat{F}}(z)$ и $L_{p}^{*}(z)$.

Лемма 1. Пусть $z_{0} \in C^{n}-$ произвольная точка, лежащая на единичной сфере. Тогда в полупространстве

$$
\left(\operatorname{Re}\left\langle z, \bar{z}_{0}\right\rangle\right) \leqslant L_{\hat{\boldsymbol{F}}}^{*}\left(z_{0}\right)+\varepsilon, \quad \bar{z}_{0}=\left(\bar{z}_{0}^{1}, \ldots, \bar{z}_{0}^{n}\right),
$$

суцествует выпуклое компактное множество, определяющее функиионал $F$.

Доказательство. Пусть $\varepsilon>0-$ произвольное сколь угодно малое писло. В пространстве $C^{n}$ возьмем замкнутый шар $B_{r}$ радиуса $r$ и построим множество:

$$
K_{r}=B_{r} \cap\left\{z \in C^{n}:\left(\operatorname{Re}\left\langle z, \bar{z}_{0}\right\rangle\right) \leqslant L_{\hat{\boldsymbol{F}}}^{*}\left(z_{0}\right)+\varepsilon\right\} .
$$

Если число $r$ достаточно велико, то можсно утверждать, что 
1) множество $K_{r}$ не пусто;

2) опорная функция $H_{K_{r}}(z)$ выпуклого множества $K_{r}$ удовлетворяет условию:

$$
L_{\hat{\mathbf{F}}}^{*}(z)<H_{\mathbf{K}_{r}}(z)+\varepsilon|z|, \quad z \in \boldsymbol{C}^{n} .
$$

Используя принцип Фрагмена-Линделёфа для субгармонических функций (см. [11], стр. 116), из (4) вытекает существование такого $r_{0}$, что неравенство

$$
L_{\hat{f}}^{*}(z)<H_{K_{r_{0}}}(z)+\varepsilon_{1}|z|, \quad z \in C^{n},
$$

вьтолняется для всякого $\varepsilon_{1}>0$. Из неравенства (5) и свойства (3) регуляризованного индикатора слэдует, что вытолняется неравенство:

$$
\ln |\hat{F}(z)|<H_{K_{r_{0}}}(z)+\varepsilon_{1}|z|,
$$

где $\varepsilon_{1}>0$ - произвольное сколь угодно малое число, $|z|>r^{\circ}\left(\varepsilon_{1}\right)$. По теореме А из последнего неравенства получаем утверждение леммы 1.

Замечание. Из доказательства леммы 1 следует, что $L_{\hat{F}}^{*}\left(z_{0}\right) \leqslant H_{\boldsymbol{K}_{0}}\left(z_{0}\right)$.

Пусть точка $z_{0}$ такая же как и в лемме 1 . Полупространство

$$
\left(\operatorname{Re}\left\langle z, \bar{z}_{0}\right\rangle\right)<L_{\hat{F}}^{*}\left(z_{0}\right)
$$

будем в дальнейшем обозначать через $\Pi\left(z_{0}\right)$.

Рассмотрим теперь полупространство $D\left(z_{0}\right)$ вида $\left(\operatorname{Re}\left\langle z, \bar{z}_{0}\right\rangle\right)<c$, где $c$ - некоторое действительное число и построим область $G\left(z_{0}\right)=D\left(z_{0}\right)+$ $+\Pi\left(z_{0}\right)$. В пространстве $H\left(G\left(z_{0}\right)\right)$ рассмотрим оператор свертки

$$
M[f]=\langle F, f(z+u)\rangle, \quad f(z) \in H\left(G\left(z_{0}\right)\right) .
$$

Из леммы 1 вытекает, что $M[f](z) \in H\left(D\left(z_{0}\right)\right)$. Изучим вопрос о том, когда оператор $M$ сорьективно отображает пространство $H\left(G\left(z_{0}\right)\right)$ на $H\left(D\left(z_{0}\right)\right)$, т.е. когда уравнение

$$
\langle F, f(z+u)\rangle=g(z)
$$

имеет решение $f(z) \in H\left(G\left(z_{0}\right)\right)$ для всякой функции $g(z) \in H\left(D\left(z_{0}\right)\right)$. Без ограничения общности можно считать, что $\hat{F}(0) \neq 0$. Введем пространства $P_{D\left(z_{0}\right)}$ и $P_{G\left(z_{0}\right)}$. Очевидно, оператор (6) является линейным и непрерывным оператором из пространства $H\left(G\left(z_{0}\right)\right)$ в $H\left(D\left(z_{0}\right)\right)$. Тогда оператор $M^{*}$, сопряженшый с $M$, является линейным и непрерывным оператором, действующим из пространства $H^{*}\left(D\left(z_{0}\right)\right)$ в $H^{*}\left(G\left(z_{0}\right)\right)$. Пусть фунгсиионал $S \in H^{*}\left(D\left(z_{0}\right)\right)$. Найдем преобразование Лапласа элемента $M^{*}[S] \in H^{*}\left[G\left(z_{0}\right)\right]$. Из определения сопряженной операции имеем:

$$
\left\langle M^{*}[S], \exp \langle\lambda, z\rangle\right\rangle=\langle S, M[\exp \langle\lambda, z\rangle]\rangle=\hat{F}(\lambda) \cdot \hat{S}(\lambda) .
$$

Отскда вытекает, что оператор $M$ порождает оператор $Z_{\hat{F}}: P_{D\left(z_{0}\right)} \rightarrow P_{G\left(z_{0}\right)}$ умножения на характеристическую функцию оператора (6). Из определения топологи в пространствах $P_{D\left(z_{0}\right)}$ и $P_{G\left(z_{0}\right)}$ следует, что оператор $Z_{\hat{F}}$ в топологическом смысле эквивалентен оператору $M^{*}$. Образ $\operatorname{Im} M$ оператора $M$ является плотным подмножеством в $H\left(D\left(z_{0}\right)\right)$. Действительно, если в уравнении (7) взять в качестве функции $g(z)$ произвольный многочлен, то методом неопределенных коэффициентов можно найти многочлен, удовлетворяющий этому уравнению (здесь используется то, что $\hat{F}(0) \neq 0)$. Так как всякое полупространство является областью Рунге, то $\overline{\operatorname{Im} M}=H\left(D\left(z_{0}\right)\right)$. Таким образом, чтобы доказать резрешимость уравнения (7) в пространстве $H\left(G\left(z_{0}\right)\right)$ для произвольной фунжции $g(z) \in H\left(D\left(z_{0}\right)\right)$ нам нужно, в силу результата Дведонне-Шварца о прямой и сопряженной операциях в пространствах типа $\mathscr{F}[12]$, выяснить, когда образ оператора $M^{*}$ является замкнутым подмножеством в $H^{*}\left(G\left(z_{0}\right)\right)$. Но последняя задача эквивалентна задаче о замкнутости множества $\operatorname{Im} Z_{\hat{F}}$ в $P_{G\left(z_{0}\right)}$.

Tворема 2. Множество $\operatorname{Im} Z_{\hat{F}}$ замкнуто в $P_{G\left(z_{0}\right)}$ mогда $u$ только тогда, когда для вслкой челой функуии экспоненчиального типа $\psi(z)$ выполняется равенство

$$
L_{\hat{F} \cdot \psi}^{*}\left(z_{0}\right)=L_{\hat{F}}^{*}\left(z_{0}\right)+L_{\psi}^{*}\left(z_{0}\right) .
$$

Доказательство. Достаточность. Пусть выполняется равенство (8) и пусть функция $N(\lambda) \in P_{G\left(z_{0}\right)}$ принадлежит замьпканию множества $\operatorname{Im} Z_{\hat{F}}$. Поскольку топология пространства $P_{G\left(z_{0}\right)}$ сильнее топологии равномерной сходимости на компактах из $C^{n}$, то функция $N(\lambda)$ делится на $\hat{F}(\lambda)$. В работе [1] показано, что функция $\psi(\lambda)=N(\lambda) / \hat{F}(\lambda)$ имеет экспоненциальньй рост. Так как вьполняется равенство (8), то $L_{w}^{*}\left(z_{0}\right)=L_{N}^{*}\left(z_{0}\right)-L_{\hat{F}}^{*}\left(z_{0}\right)$. Поскольку функция $N(\lambda)$ принадлежит $P_{G\left(z_{0}\right)}$, то существует такой выпуклый компакт $K_{N} \subset G\left(z_{0}\right)$, что

$$
|N(\lambda)|<c_{1}(\varepsilon)\left[H_{K_{N}}(\lambda)+\varepsilon|\lambda|\right], \quad \lambda \in C^{n}, \forall \varepsilon>0,
$$

и, следовательно, имеет место неравенство $L_{\psi}^{*}\left(z_{0}\right) \leqslant H_{\boldsymbol{K}_{N}}\left(z_{0}\right)-L_{\hat{\boldsymbol{F}}}^{*}\left(z_{0}\right)$. Далее, в силу замечания после леммы 1 найдется такой компакт $K_{\hat{F}} \subset \bar{\Pi}\left(z_{0}\right)$, что $H_{\boldsymbol{K}_{\hat{\boldsymbol{F}}}}\left(z_{0}\right)=L_{\hat{\boldsymbol{F}}}^{*}\left(z_{0}\right)$. Таким образом последнее неравенство можно записать так:

$$
L_{\psi}^{*}\left(z_{0}\right) \leqslant H_{K_{N}}\left(z_{0}\right)-H_{K_{\hat{F}}}\left(z_{0}\right) .
$$

Нетрудно видеть, что в полупространстве $D\left(z_{0}\right)$ существует компакт $K_{\varphi}$, для которого $H_{K_{\varphi}}\left(z_{0}\right)=H_{K_{N}}\left(z_{0}\right)-H_{\boldsymbol{K}_{\hat{F}}}\left(z_{0}\right)$. Поэтому из неравенства (9) получаем $L_{\psi}^{*}\left(z_{0}\right) \leqslant H_{K_{\psi}}\left(z_{0}\right)$. Из последнего неравенства следует, что функция $\psi(\lambda)$ принадлежит $P_{D\left(z_{0}\right)}$ (см. доказательство леммы 1 ). Таким образом достаточность теоремы 2 доказана.

Необходимость. Пусть $\operatorname{Im} Z_{\hat{F}}-$ замкнутое множество в $P_{G\left(z_{0}\right)}$. Если через $\Pi^{0}\left(z_{0}\right)$ обозначить полупространство $\left(\operatorname{Re}\left\langle z, \bar{z}_{0}\right\rangle\right)<0$, то, как нетрудно

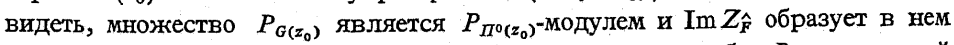
замкнутый подмодуль. Подмодуль $\operatorname{Im} Z_{\hat{F}}$ порождает в алгебре $P_{C^{n}}$ некоторый идеал, который, очевидно, являе̨тся главным с образующей $\hat{F}(\lambda)$. По тео- 
реме 3.2 работы [5] подмодуль $\operatorname{Im} Z_{\hat{F}}$ однозначно определяется своими локальньги идеалами. Отсюда следует важный для дальнейших рассуждений вывод: в подмодуль $\operatorname{Im} Z_{\hat{F}}$ входят все функции из $P_{G\left(z_{0}\right)}$, которые делятся на $\hat{F}(\lambda)$.

Пусть функция $N(\lambda) \in \operatorname{Im} Z_{\hat{F}}$. Тогда $N(\lambda)=\hat{F}(\lambda) \cdot \psi(\lambda)$, где $\psi(\lambda) \in P_{D\left(z_{0}\right)}$. Докажем, что

$$
L_{N}^{*}\left(z_{0}\right)=L_{\hat{F}}^{*}\left(z_{0}\right)+L_{\psi}^{*}\left(z_{0}\right) .
$$

Действительно, пусть имеет место строгое неравенство $L_{N}^{*}\left(z_{0}\right)<L_{\hat{F}}^{*}\left(z_{0}\right)+$ $+L_{\psi}^{*}\left(z_{0}\right)$. Тогда, как нетрудно видеть, найдется точка $t^{0} \in C^{n}$ такая, что $\psi(\lambda) \exp \left\langle t^{0}, \lambda\right\rangle \notin P_{D\left(z_{0}\right)}, \quad N(\lambda) \exp \left\langle t^{0}, \lambda\right\rangle \in P_{G\left(z_{0}\right)}$. Так как функция $N(\lambda) \exp \left\langle t^{0}, \lambda\right\rangle$ делится на $\hat{F}(\lambda)$, то $N(\lambda) \exp \left\langle t^{0}, \lambda\right\rangle \in \operatorname{Im} Z_{\hat{F}}$. Поэтому из равенства $N(\lambda) \exp \left\langle t^{0}, \lambda\right\rangle=\hat{F}(\hat{\lambda}) M(\lambda) \exp \left\langle t^{0}, \lambda\right\rangle$ должно вытекать, что $M(\lambda) \exp \left\langle t^{0}, \lambda\right\rangle \in P_{D\left(z_{0}\right)}$, что противоречит предположению относительно точки $t^{0}$. Таким образом равенство (10) доказано.

Следствив 3. Для того чтобы уравнение (7) имело решение в пространстве $H\left(G\left(z_{0}\right)\right)$ для произвольной функиии $g(z) \in H\left(D\left(z_{0}\right)\right)$ необходимо и достаточно, чтобы для всякой челой функиии экспоненциального типа $\psi(\lambda)$ выполнялось равенство (8).

\section{4. Приложения}

Пусть функционал $F \in H^{*}\left(C^{n}\right)$ таков, что

$$
L_{\hat{F}}^{*}(z)=H_{K}(z)
$$

где $H_{K}(z)$ - опорная функция некоторого выпуклого замкнутого множества $K \subset C^{n}$. Существование таких функционалов следует из результата Мартино (см. [11], стр. 294). Пусть $\mathscr{D} \subset C^{n}-$ произвольная выпуклая область. Построим область $G=\mathscr{D}+K$. В этом пункте мы будем рассматривать уравнение (7), где $g(z) \in H(\mathscr{D})$. Неоднородное уравнение свертки с условием (11) ранее рассматривалось В. В. Моржаковым [8]. Мы найдем необходимые и достаточные условия существования решения уравнения (7) для любой выпуклой области $\mathscr{D}$ и любой функции $g(z) \in H(\mathscr{D})$. Точно так же как и выше (п. 3) показывается, что данная задача эквивалентна задаче о замкнутости множества значений оператора $Z_{\hat{F}}: P_{\mathscr{D}} \rightarrow P_{G}$, умножения на функцию $\hat{F}(\lambda)$.

Творема 4. Для того итобы уравнение (7) имело реикеие в пространстве $H(G), G=\mathscr{D}+K$, для вслкой выпуклой области $\mathscr{D} u$ любой функиии $g(z) \epsilon$ $\in H(\mathscr{D})$ необходино и достаточно, чтобы выполнялось равенство (8) для любой точки $z_{0}$ и любой челой функиии экспоненциального типа $\psi(\lambda)$.

Доказательство. Необходимость условий вытекает из следствия 3, так как в качестве области $\mathscr{D}$ можно взять любое полупространство. Достаточность теоремы 4 фактически доказана в работе [8]. Пусть $\psi(\lambda) \in P_{G}$ такова, что отношение $\psi(\lambda) / \hat{F}(\lambda)$ есть целая функция. Обозначим через $S$ функционал из пространства $H^{*}(G)$, преобразование Лапласса которого равно $\psi(\lambda)$. Очевидно существует такой выпуклый компакт $K_{1} \subset \mathscr{D}$, что сумма $K_{1}+K$ является компактом, определяющим функционал $S$. Из равенства (8) следует, чTO

$$
L_{\psi(\lambda) / \hat{F}(\lambda)}^{*}\left(z_{0}\right)=L_{\psi}^{*}\left(z_{0}\right)-L_{\hat{F}}^{*}\left(z_{0}\right) \leqslant H_{K_{1}+K}\left(z_{0}\right)-H_{K}\left(z_{0}\right)=H_{K_{1}}\left(z_{0}\right) .
$$

Из последнего неравенства и теоремы А следует, что функция $\psi(\lambda) / \hat{F}(\lambda)$ принадлежит пространству $P_{\mathscr{D}}$. Таким образом, оператор $Z_{\hat{F}}$ имеет замкнутый образ в пространстве $P_{G}$ и поэтому уравнение (7) разрешимо в пространстве $H(G)$ для всякой функции $g(z) \in H(\mathscr{D})$. Поскольку область $\mathscr{D}$ в данньх рассуждениях была произвольной, то достаточность доказана. Теорема 4 доказана.

Отметим, что равенство (8) выполняется, если функция $\hat{F}(\lambda)$ имеет вполне регулярный рост в $\boldsymbol{C}^{n}$ (см. [8]).

Целую функцию $\varphi(\lambda), \lambda \in C^{n}$, имеющую экспоненциальный рост, называют (см. [12]) функцией вполне регулярного роста в $C^{n}$, если почти для всех $\lambda \in C^{n}$ выполняется равенство

$$
\lim _{\substack{r \rightarrow \infty \\ r \notin E_{0}}} \frac{1}{r} \ln |\varphi(r \cdot \lambda)|=L_{\varphi}(\lambda),
$$

где $E_{0}$ - множество относительной меры нуль, зависящее, вообще говоря, от точки $\lambda$.

Рассмотрим теперь случай размерности $n=1$. Целые функции одного комплексного переменного, имеющие вполне регулярный рост, обладают следующим свойством: если в произведении $g(\lambda) \cdot \varphi(\lambda)$ двух целых функций экспоненциального типа хотя бы один из сомножителей имеет вполне регулярный рост вдоль луча $\arg \lambda=\theta$, то для индикатрис этих функщй выполняется равенство

$$
h_{g \cdot \varphi}(\theta)=h_{g}(\theta)+h_{\varphi}(\theta) .
$$

Заметим, что в общем случае имеет место неравенство:

$$
h_{g \cdot \varphi}(\theta) \leqslant h_{g}(\theta)+h_{\varphi}(\theta) \text {. }
$$

В работе [13] В. С. Азарин доказал, что указанное выше свойство функций регулярного роста является характеристическим, т.е. имеет место следующая

Теорема В. Если для всякой челой функиии $g(\lambda)$ экспоненииального типа выполняется равенство (12), то функиия $\varphi(\lambda)$ имеет вполне регулярный рост.

Так как для целой функции $\varphi(\lambda)$ экспоненциального типа, зависящий от одного комплексного переменного, справедливо равенство

$$
L_{\varphi}(z)=L_{\varphi}^{*}(z)=h_{\varphi}(\theta) \cdot|z|, \quad \theta=\arg z, 0<\theta \leqslant 2 \pi,
$$

то из теорем 4 и В вытекает (см. [9]) 
Теорема 5. Для того чтобы уравнение (7) имело решетие в пространстве WARSAW 1983

$H(G), G=\mathscr{D}+K$, для всякой плоской выпуклой области $\mathscr{D}$ и любой функчии $g(z) \in H(\mathscr{D})$ необходимо и достаточно, чтобы функчия $\tilde{F}(\lambda)$ была функчией вполне регулярного роста в $\boldsymbol{C}$.

Отметим, что в работе [14] находятся необходимые и достаточные условия размерности уравнения (7) в пространстве $H(G), G=\mathscr{D}+K$, где $\mathscr{D}-$ фиксированная выпуклая область в плоскости $C$.

\section{Литература}

[1] B. Mal gra ng e, Existence et approximation des solution des equations aux derivees partielles et des equation de convolution, Ann. Inst. Fourier 6 (1956), 271-355.

[2] L. Ehren preis, Mean periodic functions, Amer. J. Math. 77 (1955), 293-326.

[3] A. M a r t i n e a u, Equations differentielles d'ordre infini, Bull. Soc. Math. France 95 (1967), 109-154.

[4] D. Pis a nelli, Une generalization des théoremes de convolution, C. R. Acad. Sci. Paris, A 275 (1972), 1319-1322.

[5] В. В. Н а п а лк ов, О подпространствах аналитических функиий, инвариантных относительно сдвига, Изв. АН СССР, серия матем. 36 (1972), 1269-1281.

[6] -, Уравнения типа свертки в трубчатых областях $C^{2}$, ibid. 38 (1974), 446-456.

[7] -, Об одной теореме единственности в теории функиий многих комплексных переменных u однородных уравнениях типа свертки в трубчатых областлх $C^{n}$, ibid. 40 (1976), 115-132.

[8] В. В. М о ж а ко в, Об уравнениях сверікки в пространствах функиий, голоморфных в выпукльхх областях и на выпуклых компактах в $C^{n}$, Матем. заметки 16 (1974), 431-440.

[9] В. В. Н а п а л к о в, Об одном классе неоднородных уравнений типа свертки, Успехи математич. наук 29 (1974), 217-218.

[10] Л. Хё р м а н де $\mathrm{p}$, Введение в теорию функиий нескольких комплексныхх переменных Мир, Москва 1968.

[11] Л. И. Р он к ин, Введение в теорию уельхх функиий многих переменных, Наука, Москва 1971.

[12] L. Gruma n, Entire functions of several variables and their asymptotic growth, Ark. för Math. 9 (1) (1971), 141-163.

[13] В. С. А з а р ин, Об одном характериспическом свойстве функиий вполне регулярного роста в нутри угла. Сб. Теорил функий, функииональный анализ и их приложсения, 2 , Харьков 1966, 55-67.

[14] О. В. Е п и Ф а н О в, Об эпиморфизме свертки в выпуклых областлх, Докл. АН СССР 217 (1974), 18-19.

Presented to the Semester

COMPLEX ANALYSIS

February 15-May 30, 1979

\section{SUR LES FONCTIONS ANALYTIQUES $f(x, y)$ DONT L'ENSEMBLE} DES ZÉROS PAR RAPPORT A $y$ EST ALGÉBRIQUE

\author{
NGUYEN THANH VAN \\ UER de Mathématiques, Informatique et Gestion \\ Université Paul Sabatier
}

118, Route de Narbonne, 31077-Toulouse Cedex, France

\section{Introduction. Résumé}

Dans une note parue aux C. R. A. S. [4], nous avons établi le résultat suivant

THÉORÈME 1. Soit $\Omega$ un domaine dans $C^{m}$ et $f(x, y)$ une fonction analytique sur $\Omega \times C^{n}$, pour tout $x \in \Omega$ on pose $Z_{x, f}=\left\{y \in C^{n}: f(x, y)=0\right\}$. Si l'ensemble $E_{f}=\left\{x \in \Omega: Z_{x, f}\right.$ est algébrique $\}$ n'est pas polaire dans un domaine $\Omega_{0} \Subset \Omega$, alors il existe une fonction $h$ analytique sur $\Omega \times C^{n}$ et des fonctions $A_{\alpha}\left(\alpha \in N^{n},|\alpha| \leqslant q\right)$ analytiques sur $\Omega$ telles que

$$
f(x, y)=\left(\sum_{|\alpha| \leqslant q} A_{\alpha}(x) \cdot y^{\alpha}\right) \exp h(x, y)
$$

Dans cet énoncé on peut mettre „non polaire dans $\Omega^{\prime \prime}$ à la place de „n'est pas polaire dans un domaine $\Omega_{0} \Subset \Omega^{\prime \prime}$, en raison d'un important résultat de Josefson [2]. Le but de ce travail est d'étendre cet énoncé aux fonctions analytiques sur des espaces vectoriels topologiques de Baire.

Soient $E$ et $F$ deux e.v.t. complexes séparés et de Baire dont le produit $E \times F$ est également supposé de Baire. Soit $\Omega$ un domaine dans $E$.

DÉfinitron. Un ensemble $X \subset E$ est dit finiment polaire dans $\Omega$ si et seulement si $X \neq \Omega$ et pour tout sous-espace affine de dimension finie $\Delta$ dans $E$, on a sur chaque composante connexe $C$ de $\Omega \cap \Delta$ :

- ou bien $X \supset C$

- ou bien $X \cap \Delta$ est polaire dans $C$.

Il résulte immédiatement de cette définition que si $\left(X_{n}\right)$ est une suite d'ensembles finiment polaires dans $\Omega$ et si $\bigcup_{n} X_{n} \neq \Omega$, alors $\bigcup_{n} X_{n}$ est finiment polaire dans $\Omega$.

THÉORÈME 2. Soit $f(x, y)$ une fonction analytique sur $\Omega \times F$, on pose

$$
Z_{x, f}=\{y \in F: f(x, y)=0\}
$$

